\title{
Neuroprotection of desferrioxamine in lipopolysaccharide-induced nigrostriatal dopamine neuron degeneration
}

\author{
ZHEN ZHANG $^{1}$, KEKE ZHANG ${ }^{2}$, XINRUI DU ${ }^{1}$ and YANBIN LI ${ }^{1}$ \\ Departments of ${ }^{1}$ Neurosurgery, and ${ }^{2}$ Otorhinolaryngology Head and Neck Surgery, \\ Provincial Hospital Affiliated to Shandong University, Jinan 250021, P.R. China
}

Received August 17, 2011; Accepted October 31, 2011

DOI: $10.3892 / \mathrm{mmr} .2011 .671$

\begin{abstract}
Inflammation and iron accumulation in the substantia nigra $(\mathrm{SN})$ are implicated in the pathogenesis of Parkinson's disease (PD). However, the relationship between neuroinflammation and iron mismanagement remain largely unknown. In the present study, an animal model induced by lipopolysaccharide (LPS) was used to evaluate iron concentration in the ventral midbrain with or without neuroinflammation. Furthermore, the iron chelator desferrioxamine (DFO) was used to explore its neuroprotective property against LPS-induced nigrostriatal degeneration. Adult C57BL/6 mice were treated with DFO $(2.5 \mu \mathrm{g})$ commenced 3 days prior to or following microinjection of LPS into the striatum. Animal behavioral tests, as well as pathological and biochemical assays were performed to evaluate the nigrostriatal dopamine neuron degeneration and neuroprotective effects of DFO. Here, we report that the iron concentration in the ventral midbrain significantly increased following intrastriatal injection of LPS, and administration of DFO improved behavior deficits, attenuated dopamine (DA) neuron loss and striatal DA reduction, and alleviated microglial activation in the SN. These results suggest that DFO may possess neuroprotective effect against LPS-induced nigrostriatal dopamine neuron degeneration.
\end{abstract}

\section{Introduction}

Parkinson's disease (PD) is a common neurodegenerative movement disorder, characterized by a progressive loss of dopaminergic (DA) neurons in the substantia nigra (SN), striatal dopamine depletion (1) and behavioral impairments (2). Numerous lines of study have shown that aberrant iron accumulation in the SN plays a pivotal role in the pathogenesis of PD $(3,4)$. The major mechanism of iron-mismanagement was related to increased iron uptake by DMT1, Tf/TfR2 and Lf/

Correspondence to: Dr Keke Zhang, Department of Otorhinolaryngology Head and Neck Surgery, Provincial Hospital Affiliated to Shandong University, 324 Jingwuweiqi Road, Jinan 250021, P.R. China

E-mail: zhangzhen312@gmail.com

Key words: Parkinson's disease, lipopolysaccharide, desferrioxamine, inflammation
LfR (5-7), decreased iron export by Fp1 and Cp $(8,9)$, and iron storage misregulation by ferritin and lysosome (10-12). Application of iron chelation demonstrated a protective effect against DA neuron degeneration in PD animal models induced by N-methyl-4-pheny-1, 2, 3, 6-tetrahydropyridine (MPTP) and lactacystin (4,13-15).

Evidence also suggests that chronic inflammation is involved in the pathogenesis of PD (16). As an inflammogen, lipopolysaccharide (LPS) has been demonstrated as an effective initiator of nigrostriatal DA neuronal death in experimental animals (17). Recently, a PD model was created via intrastriatal LPS injection in C57BL/6 mice, revealing loss of tyrosine hydroxylase (TH)-positive cells, progressive dopaminergic neuron death in the $\mathrm{SN}$, progressive decrease of behavioral performance and striatal dopamine decrease (18). It appears that injection of LPS into the striatum may become a suitable model for revealing the potential role of inflammation in PD-related neurodegeneration.

The primary goal of the proposed study was to investigate whether iron accumulation existed in the ventral midbrain of C57BL/6 mice following intrastriatal injection with LPS and to determine whether iron chelator desferrioxamine (DFO) possesses neuroprotective properties against LPS-induced nigrostriatal degeneration. The results of the study may provide new insight into the potential novel mechanisms for the treatment of PD.

\section{Materials and methods}

Animals and treatments. The proposed animal study was approved by the Ethics Committee of Shandong University. Male C57BL/6 mice aged 12 weeks were randomly signed into four groups: control, LPS, pre-DFO and post-DFO. Intracerebroventricular (ICV) administration of DFO $(2.5 \mu \mathrm{g}$; Sigma, St. Louis, MO, USA) was commenced 3 days prior to or following microinjection of LPS, while the administration of the same volume of saline served as control. For stereotactic injection of LPS and DFO, mice were anesthetized by Avertin (200 mg/kg, intraperitoneal injection) and placed on a stereotactic apparatus (Kopf Instruments, Tujunga, CA, USA). Injection was performed through drilled holes in the skull, into the striatum or paracele using the following coordinate (in $\mathrm{mm}$ ): 0.5 posterior, \pm 2.1 lateral and 3.8 ventral from bregma for striatum (19), 0.5 posterior, \pm 1.0 lateral and 2.0 ventral 

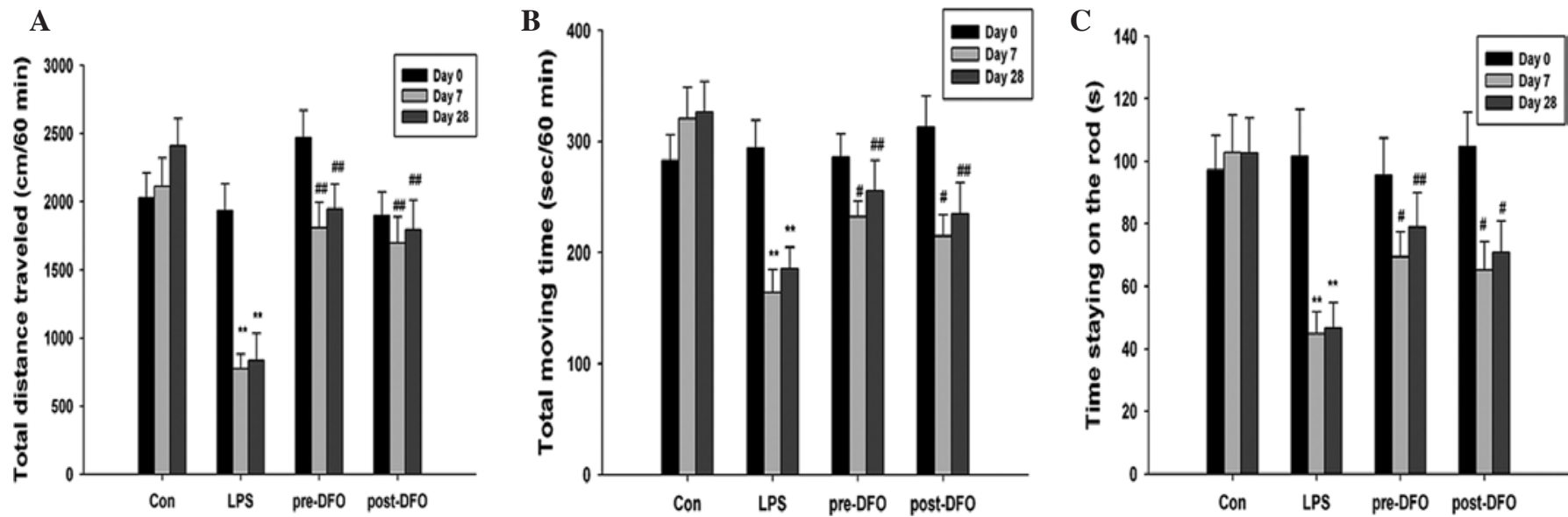

Figure 1. DFO improves behavioral performance in LPS-lesioned mice. Locomotive activities and rotarod performance were performed 1 day prior to (set to be day 0 ), and 7 and 28 days following microinjection of LPS. Changes in locomotive activity are shown by (A) total distance traveled and (B) moving time. (C) The alteration of rotarod performance was recorded by time staying on the rod. Data were expressed as the means $\pm \mathrm{SE}$ ( $\mathrm{n}=5$ ). ${ }^{* *} \mathrm{P}<0.01 \mathrm{vs}$. control; ${ }^{\#} \mathrm{P}<0.05$ vs. LPS, day 28; ${ }^{\#} \mathrm{P}<0.01$ vs. LPS, day 28. LPS, lipopolysaccharide; SE, standard error; DFO, desferrioxamine; Con, control.

from bregma for DFO. A total of $2 \mu 1$ of phosphate-buffered saline (PBS; $0.1 \mathrm{M}$ ) as control or LPS (10 $\mu \mathrm{g}$; Sigma) in PBS was injected into the striatum of each mouse.

All mice were sacrificed by $\mathrm{CO}_{2} 28$ days following administration of LPS, followed by transcardial perfusion with ice-cold PBS. The right midbrains of these mice were immediately removed, fixed in $4 \%$ paraformaldehyde for $48 \mathrm{~h}$ and cryoprotected in $30 \%$ sucrose for $48 \mathrm{~h}$ at $4^{\circ} \mathrm{C}$, followed by histological analysis. Striatal tissues and left ventral midbrains were rapidly dissected out and stored at $-80^{\circ} \mathrm{C}$ until analysis.

Locomotion activities and rotarod performance. Locomotive activities and rotarod performance were examined 1 day prior to (set to be day 0 ), and 7 and 28 days following microinjection of LPS. For the locomotor test, the animal was placed in a chamber $(40 \times 40 \times 30 \mathrm{~cm})$ and locomotion activities were recorded in a 60-min section using the AccuScan Digiscan system (AccuScan Instruments, Inc., Columbus, OH, USA), including total distance and moving time. Data were generated automatically by a computer-aided monitoring system. Rotarod performance was determined with a rotarod treadmill (Columbus Instruments, Columbus, OH, USA). The mice were placed on the stationary rod for $30 \mathrm{sec}$ for acclimatization. First, a constant speed of $5 \mathrm{rpm}$ for $90 \mathrm{sec}$ was used for pretraining. Then, mice were tested three times at 1 -h intervals on 3 consecutive days for a total of nine tests. During each test, the rotarod began at a speed of $5 \mathrm{rpm}$ for $30 \mathrm{sec}$, followed by an increase of $0.1 \mathrm{rpm} / \mathrm{sec}$.

Immunohistochemistry. Coronal sections (30- $\mu \mathrm{m})$ were cut through the entire SN using a Microm HM550 cryostat (Germany). Immunohistochemistry was performed as previously described by Li et al (20). Briefly, sections were incubated in $0.05 \% \mathrm{H}_{2} \mathrm{O}_{2}$ in $0.1 \mathrm{M}$ PBS for 20 min to block endogenous peroxidase, in a $2 \%$ goat serum $/ 0.1 \%$ Triton $\mathrm{X}-100$ in $0.1 \mathrm{M}$ PBS for $1 \mathrm{~h}$ to block non-specific binding sites. Then, the sections were incubated in the primary antibodies, rabbit anti-tyrosine hydroxylase (TH) (1:1,500; Protos Biotech, USA) to detect DA neurons; rat anti-CD11b (against MAC1, 1:50; Chemicon International Inc., Billerica, MA, USA) to label microglia at $4^{\circ} \mathrm{C}$ overnight. Following that, the sections were incubated with the appropriate secondary antibody (anti-rabbit or anti-rat IgG, 1:200; Vector Laboratories Inc., Burlingame, CA, USA) for $2 \mathrm{~h}$ at room temperature. The avidin-biotin method was used to amplify the signal (ABC kit; Vector Laboratories Inc.), and 3,3-diaminobenzidine tetrachloride (DAB) was used to visualize bound antibodies.

Determination of striatal DA and its metabolites. The concentrations of DA, 4-dihydroxy-phenylacetic acid (DOPAC) homovanillic acid (HVA) in striatal tissues were quantified by HPLC. Briefly, striatal tissues mixed with ice-cold $0.1 \mathrm{M}$ perchloric acid were homogenized by sonication, followed by centrifuging at $14,000 \mathrm{x} \mathrm{g}$ for $12 \mathrm{~min}$ at $4^{\circ} \mathrm{C}$. The supernatants were filtered through acrodisc filters $(0.25-\mu \mathrm{m})$, subjected to HPLC (HTEC-500; Eicom, Japan) with the column (EICOM-PAK SC-3ODS; Eicom) and detected by an electrochemical detector (AD Instruments Pty Ltd., Castle Hill, Australia). The mobile phase $(\mathrm{pH} 3.5)$ consisted of $0.1 \mathrm{mM}$ citric acid, $0.1 \mathrm{M}$ sodium acetate, $220 \mathrm{mg} / \mathrm{l}$ octane sulfate sodium, $5 \mathrm{mg} / \mathrm{l}$ EDTA and $20 \%$ methanol.

Iron measurement in the ventral midbrain. Wet ventral midbrains were weighed and then digested in concentrated hydrochloric acid. Tissue iron concentrations ( $\mathrm{nmol} / \mathrm{g}$ ) were determined spectrophotometrically using the kit from DCL (Diagnostic Chemicals Ltd., Oxford, CT, USA) in a modified microtiter plate assay as previously described by Zhu et al (14).

Statistical analysis. Data were analyzed using SPSS 13.0 software (SPSS Inc., Chicago, IL, USA). The mean values among 4 groups were analyzed by using one-way analyses of variance (ANOVA) test, followed by post hoc Dunn's multiple comparisons analysis. P-values of $<0.05$ were considered to denote statistical significance.

\section{Results}

DFO improved behavioral performance in LPS-lesioned mice. Compared to the control, locomotive activities (total distance 
Table I. Concentrations of DA and its metabolites in substantia nigra of the various treatment groups.

\begin{tabular}{lcccc}
\hline $\begin{array}{l}\text { Catecholamine } \\
\text { mg) }\end{array}$ & Control $(\mathrm{ng} / \mathrm{mg})$ & LPS $(\mathrm{ng} / \mathrm{mg})$ & Pre-DFO $(\mathrm{ng} / \mathrm{mg})$ & Post-DFO (ng/ \\
\hline DA & & & \\
DOPAC & $6.73 \pm 0.54$ & $1.64 \pm 0.18^{\mathrm{b}}$ & $4.75 \pm 0.43^{\mathrm{d}}$ & $4.28 \pm 0.51^{\mathrm{d}}$ \\
HVA & $2.64 \pm 0.37$ & $0.85 \pm 0.12^{\mathrm{b}}$ & $1.51 \pm 0.35^{\mathrm{c}}$ & $1.36 \pm 0.28^{\mathrm{c}}$ \\
\hline
\end{tabular}

DFO restored LPS-induced depletion of DA and its metabolites. The results are expressed as the means $\pm \mathrm{SE}$. ${ }^{\mathrm{a} P}<0.05 \mathrm{vs}$. Control; ${ }^{\mathrm{b}} \mathrm{P}<0.01 \mathrm{vs}$. Control; ${ }^{\mathrm{P}}<0.05$ vs. LPS; ${ }^{\mathrm{d}} \mathrm{P}<0.01$ vs. LPS. DA, dopamine; LPS, lipopolysaccharide; DFO, desferrioxamine; DOPAC, 4-dihydroxy-phenylacetic acid; HVA, homovanillic acid; SE, standard error.

A
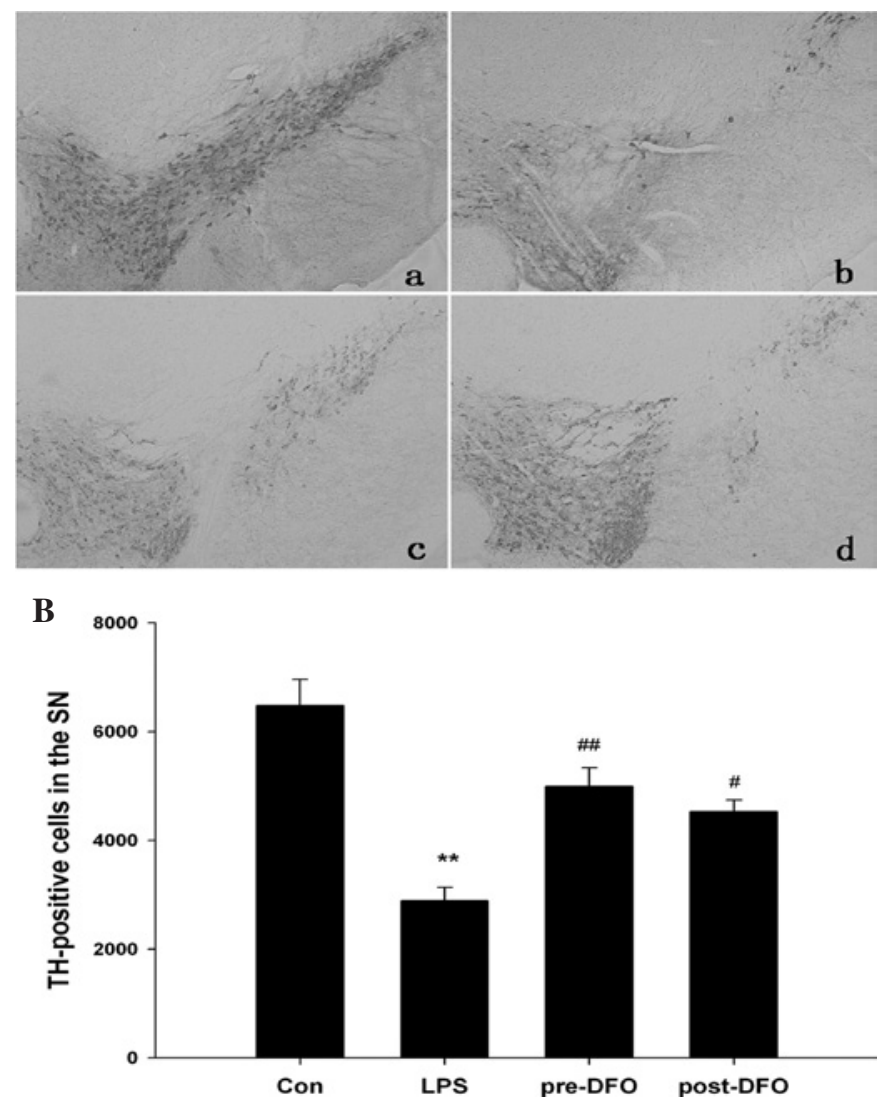

Figure 2. DFO alleviates LPS-induced loss of DA neurons in the SN. (A) Representative photomicrographs of SN with TH immunohistochemistry (magnification, x10). (a-d) Control,LPS, pre-DFO and post-DFO, respectively. (B) Quantification of TH-immunopositive neurons in the SN. Values were presented by the means $\pm \mathrm{SE}$ based on the number of TH-immunopositive neurons in the right hemispheric nigral slices $(\mathrm{n}=5)$. ${ }^{* *} \mathrm{P}<0.01$ vs. control; ${ }^{\#} \mathrm{P}<0.05$ vs. LPS, day $28 ;{ }^{\# \#} \mathrm{P}<0.01$ vs. LPS, day 28 . LPS, lipopolysaccharide; $\mathrm{SE}$, standard error; DFO, desferrioxamine; TH, tyrosine hydroxylase; SN, substantia nigra; Con, control.

traveled and moving time) of mice injected with LPS were significantly decreased by $63.3 \%(\mathrm{P}<0.01)$ and $48.9 \%(\mathrm{P}<0.01)$ on day 7 , and decreased by $65.4 \%(\mathrm{P}<0.01)$ and $43.1 \%(\mathrm{P}<0.01)$ on day 28, respectively (Fig. $1 \mathrm{~A}$ and $\mathrm{B}$ ). The rotarod performance (time staying on the rod) was reduced by $56.4 \%(\mathrm{P}<0.01)$ on day 7 and $54.5 \%(\mathrm{P}<0.01)$ on day 28 (Fig. 1C), respectively.

Pre-treatment of DFO significantly attenuated behavioral impairment by $77.3 \%(\mathrm{P}<0.01)$ and $70.6 \%(\mathrm{P}<0.01)$ in total distance traveled, by $43.6 \%(\mathrm{P}<0.05)$ and $49.6 \%(\mathrm{P}<0.01)$ in moving time, and by $42.5 \%(\mathrm{P}<0.05)$ and $57.5 \%(\mathrm{P}<0.01)$ in rotarod time, respectively, on days 7 and 28. Recovery was observed on the mice post-treated with DFO, 69.0\% $(\mathrm{P}<0.01)$ and $61.0 \%(\mathrm{P}<0.01)$ of control in total distance traveled, $32.6 \%$ $(\mathrm{P}<0.05)$ and $34.8 \%(\mathrm{P}<0.01)$ of control in moving time, $35.2 \%$ $(\mathrm{P}<0.05)$ and $43.1 \%(\mathrm{P}<0.05)$ of control in rotarod time on days 7 and 28 , respectively.

Effects of DFO against LPS-induced DA neuron loss in SN. Compared to the control, the number of DA neurons in the $\mathrm{SN}$ was reduced in LPS-lesioned mice by $55.4 \%(\mathrm{P}<0.01)$. Pre-treatment with DFO markedly protected the DA neurons in SN against LPS-induced injury by $47.1 \%(\mathrm{P}<0.01)$ and post-treatment of DFO significantly restored DA neuron degeneration by $37.4 \%(\mathrm{P}<0.05)$ (Fig. 2A and $\mathrm{B})$.

Effects of DFO against LPS-induced depletion of DA and its metabolites in SN. A loss of $75.6 \%$ DA content was traced in the striatum following microinjection of LPS compared with the control $(\mathrm{P}<0.01)$. Pre-treatment with DFO significantly protected striatal DA by $61.1 \%(\mathrm{P}<0.01)$. Compared to the control, DOPAC and HVA levels were decreased by $67.8 \%(\mathrm{P}<0.01)$ and $55.8 \%(\mathrm{P}<0.05)$ in LPS-lesioned mice. Pre-treatment with DFO showed reduction in the loss of DOPAC and HVA by $36.9 \%(\mathrm{P}<0.05)$ and $53.4 \%(\mathrm{P}<0.01)$, respectively. Post-treatment with DFO significantly restored DA by $51.9 \%(\mathrm{P}<0.01)$, DOPAC by $28.5 \%(\mathrm{P}<0.05)$ and HVA by $39.7 \%(\mathrm{P}<0.05)$ (Table I).

DFO alleviates microglia activation in LPS-lesioned mice. Microglia activation was detected by CD11b staining and morphological characterization. Compared to the vehicle control, approximately a 1.6-fold increase in microglial profile was observed in the SN of mice injected with LPS. A dense deposition of hypertrophic microglia was observed in the CD11b immunostaining profiles (Fig. 3A). Compared to microinjection of LPS, pre- and post-treatment with DFO significantly inhibited microglia activation by $57.8 \%(\mathrm{P}<0.01)$ and 51.6\% $(\mathrm{P}<0.05)$, respectively (Fig. 3B).

DFO attenuates LPS-induced iron accumulation. Iron concentration in the ventral midbrain was elevated at the end of our study, and the ability of DFO to chelate brain iron was tested in the LPS-injected mice. Compared to the vehicle control, 
A

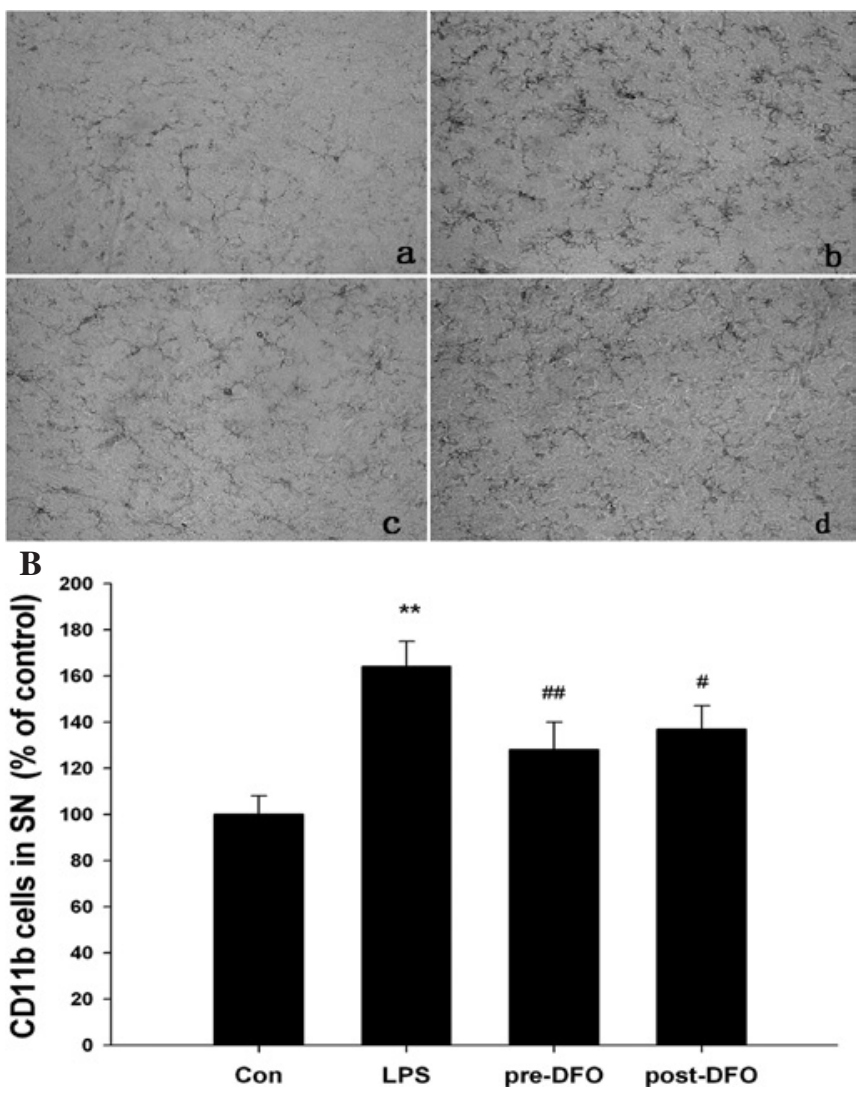

Figure 3. DFO reduces microglial activation induced by LPS. (A) The microglial profile in the SN was observed by CD11b immunohistochemistry (magnification, x40). (a-d) Control, LPS, pre-DFO and post-DFO, respectively. (B) Quantification of CD11b-positive microglia in the left SN. The results were expressed as the means $\pm \mathrm{SE}(\mathrm{n}=5)$. ${ }^{* *} \mathrm{P}<0.01$ vs. Control; ${ }^{*} \mathrm{P}<0.05$ vs. LPS, day $28 ;{ }^{\# \#} \mathrm{P}<0.01$ vs. LPS, day 28 . DFO, desferrioxamine; SN, substantia nigra; Con, control; LPS, lipopolysaccharide; SE, standard error.

iron concentration of the ventral midbrain in LPS-lesioned mice significantly increased by $44.9 \%(\mathrm{P}<0.01)$. Pre- and postapplication of DFO significantly attenuated iron overload in the ventral midbrain by $86.3 \%(\mathrm{P}<0.01)$ and $67.5 \%(\mathrm{P}<0.05)$, respectively (Fig. 4).

\section{Discussion}

Numerous studies have previously revealed that neuroinflammation plays a pivotal role in the pathogenic process of PD and may underlie the molecular mechanisms attributed to neurodegeneration in the SN (21-23). It has been demonstrated that inflammatory mediators, such as ROS, NO, TNF- $\alpha$ and interleukin (IL)- $1 \beta$ derived from non-neuronal cells, including microglia, modulate the progression of neuronal cell death in PD (24). Studies of LPS-mediated neurotoxicity provided sufficient evidence that inflammatory responses cause loss of dopaminergic neurons (25). Injection of LPS into mouse brains was found to cause increased levels of inflammatory mediators, such as COX-2 and iNOS, which may be involved in nigrostriatal neuron degeneration (26). Microglia are the primary initial responders to LPS and produce mediators, such as TNF- $\alpha$ and IL-1 $\beta$ (27). The combination of factors produced by activated microglia may promote neurotoxicity,

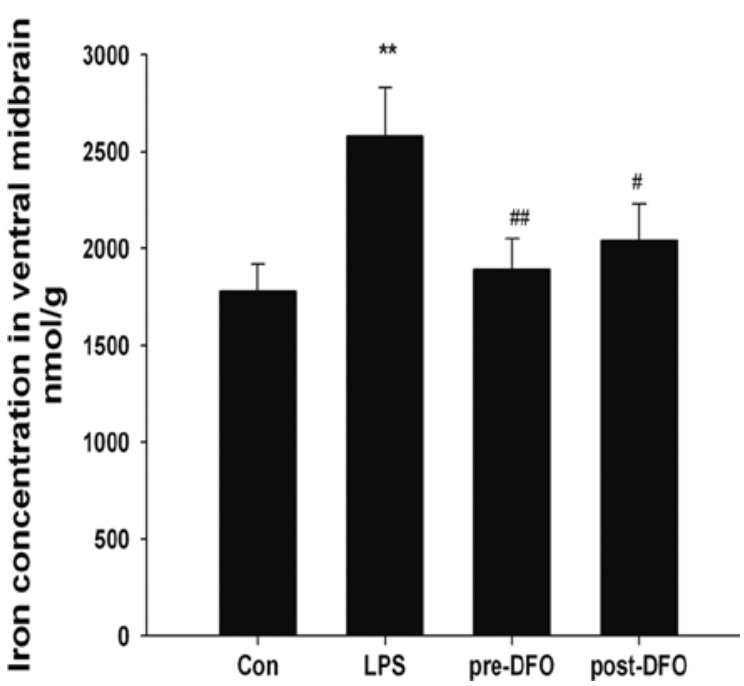

Figure 4. DFO attenuates LPS-induced iron accumulation. The results were expressed as the means $\pm \mathrm{SE}(\mathrm{n}=5) .{ }^{* *} \mathrm{P}<0.01$ vs. Control; ${ }^{\#} \mathrm{P}<0.05$ vs. LPS, day 28; ${ }^{\# \#} \mathrm{P}<0.01$ vs. LPS, day 28. DFO, desferrioxamine; Con, control; LPS, lipopolysaccharide; SE, standard error.

leading to nigrostriatal degeneration. In the present study, we modeled neuroinflammation through the application of LPS to the striatum in mice. Using this animal model, we observed significant nigrostriatal neuron degeneration, microglial activation and behavioral deficits induced by LPS, which provides us with a novel tool to study the role of neuroinflammation in PD.

Iron is the most abundant transition metal in the body, with high concentrations in the brain (28). The most common locations of high iron concentration in the brain are the globus pallidus, red nucleus and the $\mathrm{SN}$, which are most vulnerable to PD (29). Several lines of study have shown a progressive accumulation of iron in PD patients, particularly in the SN pars compacta (30). Iron-mediated DA neuron damage is related to the fact that iron is involved in the generation of reactive oxygen species (ROS). Hydrogen peroxide $\left(\mathrm{H}_{2} \mathrm{O}_{2}\right)$ is produced during oxidative deamination of DA, which reacts with $\mathrm{Fe}^{2+}$, leading to the production of hydroxyl radicals $(-\mathrm{OH})$ that damage proteins, nucleic acids and membrane phospholipids, which may cause DA neuron injury (31).

In the present study, we found that the iron concentration in the SN was significantly increased compared to the vehicle control after microinjection of LPS. One of the most possible reasons for iron accumulation may relate to the dysregulation of nigral iron homeostasis as a result of DA neuron loss and iron released from neuromelanin. Furthermore, we demonstrated that application of iron chelator DFO significantly alleviated the nigrostiatal neurodegeneration and iron accumulation and inhibited the microglial activation in the SN, indicating that DFO may be involved in the neuroprotection against neuroinflammation induced by LPS.

In conclusion, our study demonstrated that iron chelator DFO possesses protective properties against LPS-induced DA neuron death and increased iron levels. The biological effects of DFO against neuroinflammation may open a novel avenue by which to explore additional molecular mechanisms of iron chelation in the neuroprotection of PD. 


\section{References}

1. Hornykiewicz O: Parkinson's disease and the adaptive capacity of the nigrostriatal dopamine system: possible neurochemical mechanisms. Adv Neurol 60: 140-147, 1993.

2. Youdim MB and Riederer P: Understanding Parkinson's disease. Sci Am 276: 52-59, 1997.

3. Sofic E, Riederer P, Heinsen H, et al: Increased iron (III) and total iron content in post mortem substantia nigra of Parkinsonian brain. J Neural Transm 74: 199-205, 1988.

4. Kaur D, Yantiri F, Rajagopalan S, et al: Genetic or pharmacological iron chelation prevents MPTP-induced neurotoxicity in vivo: a novel therapy for Parkinson's disease. Neuron 37: 899-909, 2003.

5. Salazar J, Mena N, Hunot S, et al: Divalent metal transporter 1 (DMT1) contributes to neurodegeneration in animal models of Parkinson's disease. Proc Natl Acad Sci USA 105: 18578-18583, 2008.

6. Mastroberardino PG, Hoffman EK, Horowitz MP, et al: A novel transferrin/TfR2-mediated mitochondrial iron transport system is disrupted in Parkinson's disease. Neurobiol Dis 34: 417-431, 2009.

7. Snyder AM and Connor JR: Iron, the substantia nigra and related neurological disorders. Biochim Biophys Acta 1790: 606-614, 2009.

8. Lee DW, Rajagopalan S, Siddiq A, et al: Inhibition of prolyl hydroxylase protects against 1-methyl-4-phenyl-1,2,3,6-tetrahydropyridine-induced neurotoxicity: model for the potential involvement of the hypoxia-inducible factor pathway in Parkinson disease. J Biol Chem 284: 29065-29076, 2009.

9. Song N, Wang J, Jiang $\mathrm{H}$ and Xie J: Ferroportin 1 but not hephaestin contributes to iron accumulation in a cell model of Parkinson's disease. Free Radic Biol Med 48: 332-341, 2010.

10. Double KL and Halliday GM: New face of neuromelanin J Neural Transm 70: S119-S123, 2006.

11. Dong XP, Cheng X, Mills E, et al: The type IV mucolipidosisassociated protein TRPML1 is an endolysosomal iron release channel. Nature 455: 992-996, 2008.

12. Johansson AC, Appelqvist H, Nilsson C, Kagedal K, Roberg K and Ollinger K: Regulation of apoptosis-associated lysosomal membrane permeabilization. Apoptosis 15: 527-540, 2010.

13. Mandel S, Weinreb O, Amit T and Youdim MB: Cell signaling pathways in the neuroprotective actions of the green tea polyphenol (-)-epigallocatechin-3-gallate: implications for neurodegenerative diseases. J Neurochem 88: 1555-1569, 2004.

14. Zhu W, Xie W, Pan T, et al: Prevention and restoration of lactacystin-induced nigrostriatal dopamine neuron degeneration by novel brain-permeable iron chelators. FASEB J 21: 3835-3844, 2007.

15. Zhang X, Xie W, Qu S, Pan T, Wang X and Le W: Neuroprotection by iron chelator against proteasome inhibitor-induced nigral degeneration. Biochem Biophys Res Commun 333: 544-549, 2005 .
16. McGeer PL, Yasojima K and McGeer EG: Inflammation in Parkinson's disease. Adv Neurol 86: 83-89, 2001.

17. Kim WG, Mohney RP, Wilson B, Jeohn GH, Liu B and Hong JS: Regional difference in susceptibility to lipopolysaccharide-induced neurotoxicity in the rat brain: role of microglia. J Neurosci 20: 6309-6316, 2000.

18. Hunter RL, Cheng B, Choi DY, et al: Intrastriatal lipopolysaccharide injection induces parkinsonism in C57/B6 mice. J Neurosci Res 87: 1913-1921, 2009.

19. Calkins MJ, Jakel RJ, Johnson DA, Chan K, Kan YW and Johnson JA: Protection from mitochondrial complex II inhibition in vitro and in vivo by Nrf2-mediated transcription. Proc Natl Acad Sci USA 102: 244-249, 2005.

20. Li C, Biswas S, Li X, Dutta AK and Le W: Novel D3 dopamine receptor-preferring agonist D-264: Evidence of neuroprotective property in Parkinson's disease animal models induced by 1-methyl-4-phenyl-1,2,3,6-tetrahydropyridine and lactacystin. J Neurosci Res 88: 2513-2523, 2010.

21. Gao HM, Zhou H, Zhang F, Wilson BC, Kam W and Hong JS: HMGB1 acts on microglia Mac1 to mediate chronic neuroinflammation that drives progressive neurodegeneration. J Neurosci 31: 1081-1092, 2011.

22. Jung BD, Shin EJ, Nguyen XK, et al: Potentiation of methamphetamine neurotoxicity by intrastriatal lipopolysaccharide administration. Neurochem Int 56: 229-244, 2010.

23. Choi DY, Liu M, Hunter RL, et al: Striatal neuroinflammation promotes Parkinsonism in rats. PLoS One 4: e5482, 2009.

24. Hirsch EC and Hunot S: Neuroinflammation in Parkinson's disease: a target for neuroprotection? Lancet Neurol 8: 382-397, 2009.

25. Castano A, Herrera AJ, Cano J and Machado A: Lipopolysaccharide intranigral injection induces inflammatory reaction and damage in nigrostriatal dopaminergic system. J Neurochem 70: 1584-1592, 1998.

26. Hunter RL, Dragicevic N, Seifert K, et al: Inflammation induces mitochondrial dysfunction and dopaminergic neurodegeneration in the nigrostriatal system. J Neurochem 100: 1375-1386, 2007.

27. Saijo K, Winner B, Carson CT, et al: A Nurr1/CoREST pathway in microglia and astrocytes protects dopaminergic neurons from inflammation-induced death. Cell 137: 47-59, 2009.

28. Gotz ME, Double K, Gerlach M, Youdim MB and Riederer P: The relevance of iron in the pathogenesis of Parkinson's disease. Ann NY Acad Sci 1012: 193-208, 2004.

29. Hallgren B and Sourander P: The effect of age on the non-haemin iron in the human brain. J Neurochem 3: 41-51, 1958.

30. Berg D, Becker G, Riederer P and Riess O: Iron in neurodegenerative disorders. Neurotox Res 4: 637-653, 2002.

31. Gutteridge JM: Iron and oxygen radicals in brain. Ann Neurol 32: S16-S21, 1992. 